\title{
Perinatal and neonatal outcome in patients with preeclampsia
}

\author{
Katarzyna A. Stefanska' ${ }^{1}$, Maciej Zielinski ${ }^{2} \mathbb{D}^{-}$, Joanna Jassem-Bobowicz ${ }^{3} \mathbb{D}^{\text {, }}$

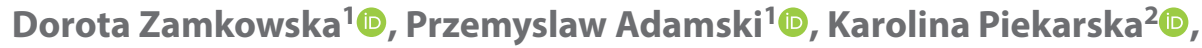 \\ Martyna Jankowiak ${ }^{2}{ }^{\mathbb{D}}$, Katarzyna Leszczynska ${ }^{1}{ }^{\mathbb{D}}$, Renata Swiatkowska-Stodulska ${ }^{4}{ }^{\mathbb{D}}$, \\ Krzysztof Preis ${ }^{1}{ }^{1}$, Piotr Trzonkowski ${ }^{2}$, Natalia Marek-Trzonkowska ${ }^{5}$ \\ ${ }^{1}$ Department of Obstetrics, Medical University of Gdansk, Poland \\ ${ }^{2}$ Department of Medical Immunology, Medical University of Gdansk, Poland \\ ${ }^{3}$ Department of Neonatology, Medical University of Gdansk, Poland \\ ${ }^{4}$ Department of Endocrinology and Internal Medicine, Medical University of Gdansk, Poland \\ 5 International Centre for Cancer Vaccine Science Cancer Immunology Group, University of Gdansk, Poland
}

\begin{abstract}
Objectives: Preeclampsia (PE) affects $2-5 \%$ of pregnant women. Hypertensive disorders of pregnancy are associated with adverse maternal and perinatal outcomes.

Material and methods: This study included 88 women showing gestational hypertension (GH) or PE symptoms, and their newborns.

Results: The rate of FGR was $43 \%$ for mothers with PE, compared to $8 \%$ with GH. The association was significant, $\mathrm{p} \leq 0.001 \mathrm{but}$ with moderate strength, Cramer's V $=0.40$. The risk of FGR increased nine times when PE occurred, as the odds ratio was 9.25 ( $\mathrm{Cl}$ : 2.46-34.83), $\mathrm{p}=0.001$. PE was associated with FGR risk if delivery time was less than 34 weeks compared to a delivery time of more than 34 weeks. This was $82 \%$ of FGR cases for $<34$ weeks, compared with $35 \%$ of cases in $>34$ group, $(p=0.001$; Cramer's $V=0.50)$. PE was also associated ( $p=0.01$, Cramer's $V=0.27)$ with the type of delivery, as the caesarean section rate was $74 \%$, compared to $50 \%$ in the $\mathrm{GH}$ group. This made it three times higher the likelihood of delivery by caesarean section, as the odds ratio was $3.10(\mathrm{Cl}: 1.24-7.75), p=0,02$. Delivery time was significantly ( $p<0.001)$ shortened to 38 weeks (27-41), compared to 40 weeks (38-42) GH mothers. There was no distinction in median age for PE and GH mothers $(p=0.124)$. The overall clinical status of neonates was proportional despite the mother's PE. The sum of Apgar points in the first, and then the second to third minute, did not differ significantly, $p=0.370$ and 0.560 , respectively. The number of peripheral blood platelets and leucocytes was not reduced $(p=0.821$ and 0.534$)$ in infants when the mother suffered from PE.
\end{abstract}

Conclusions: The prediction of adverse maternal outcomes from hypertensive diseases of pregnancy is key to optimal management, including the timing of delivery and planning for the most appropriate place of care.

Key words: preeclampsia; gestational hypertension; complications; neonates; perinatal outcome; neonatal outcome

Ginekologia Polska 2022; 93, 3: 203-208

\section{INTRODUCTION}

Preeclampsia (PE) affects $2-5 \%$ of pregnant women. It is one of the leading causes of maternal and perinatal morbidity and mortality, especially the early onset of PE [1, 2]. About 76,000 women and 500,000 babies die each year because of PE [1, 3].

According to the ISSHP PE, transient gestational hypertension and gestational hypertension $(\mathrm{GH})$ are char- acterized by the new onset of hypertension (blood pressure $\geq 140 \mathrm{mmHg}$ or $\geq 90 \mathrm{mmHg}$ ) at or after 20 weeks of gestation [4]. It is therefore important to have normal blood pressure readings documented either pre-pregnancy or in early pregnancy before there has been much pregnancy-related decrease in blood pressure [4-6].

$\mathrm{GH}$ is hypertension arising de novo after 20 weeks of gestation in the absence of proteinuria and without biochemi- 
cal or hematological abnormalities, usually not accompanied by FGR. Outcomes in pregnancies complicated solely by $\mathrm{GH}$ are generally good, however, about a quarter of women with GH (particularly those who present at $<34$ weeks) will progress to PE and have poorer outcomes [4].

There are early-onset PE (before 34 weeks of gestation) and late-onset $P E$ ( $\geq 34$ weeks gestation). Early-onset $P E$ is less prevalent but has higher rates of maternal morbidity, perinatal death and severe neonatal morbidity compared to the late-onset disease [7]. In the late-onset PE women most often have minimal or no abnormal vascular remodeling of the spiral arteries and no intrauterine FGR appears [8].

$\mathrm{PE}$ is also associated with adverse neonatal outcomes beyond morbidity caused by preterm birth. Although severe neonatal outcomes were less common at later gestational ages, there was an elevated neonatal risk due to PE even if it was possible to deliver all infants at term. Most common complications included increased perinatal mortality (OR 2.2) and an elevated risk for admission of the neonate to the intensive care unit [9].

Neonatal mortality: It has been estimated that the hypertensive disorders of pregnancy precede $10 \%$ of early neonatal deaths ( $8 / 1000$ live births) and late neonatal deaths (3/1000 live births). About 1.5-2 million neonatal deaths a year $[10,11]$ because of iatrogenic prematurity, FGR and fetal overgrowth, hypertensive disorders of pregnancy are identifiable risk factors for newborn morbidity [12].

In this study, we aimed to determine the risk of perinatal and neonatal complication in a woman with PE. Additionally, we have evaluated if the standard of care of hypertension in pregnancy is sufficient to minimize deleterious effects in newborns.

\section{MATERIAL AND METHODS}

This single-center, prospective study aimed to assess perinatal and neonatal outcomes in pregnant women burdened with GH or PE. Patients were followed up throughout the pregnancy to collect data on clinical symptoms related to hypertension. Next, the perinatal and neonatal outcome was estimated to determine whether this condition affects a newborn's healthiness. The study was supported with the Polish NCN no. 2014/15/B/NZ5/03499.

\section{Pregnant women}

Patients qualified for the study were recruited among the patients of Department of Obstetrics Medical University of Gdansk, Poland. We have recruited a cohort of pregnant woman, hospitalized between April 2015 and July 2017. Based on clinical symptoms and laboratory markers, patients were classified as gestational hypertension group $(n=44)$ and PE group ( $n=44)$ during the admission to the clinic due to exacerbation of the symptoms. The patients were hospitalized at various periods of pregnancy. The majority were admitted beyond 34th week of pregnancy. Those admitted prior to 32nd week of pregnancy were admitted due to exacerbation of hypertension, worsening of general condition of FGR. The median period of gestation differs $(\mathrm{p}<0.001)$ between $P E$ and $G H$, as it was $36(\min .27 ; \max 41)$ vs $39(\min 35 ; \max$ 42) weeks, respectively. Patients qualified for the study followed routinely obstetrical control visits as advised by the Polish standards of medical care during pregnancy. The basic information of the study population is given in Table 1.

The study inclusion criteria were 27 to 42 weeks of gestation, singleton pregnancy with GH or PE but without co-morbidities.

\section{Infants}

Infants included in the study were all born in the Department of Obstetrics, Medical University of Gdańsk. They were singleton neonates born to mothers with either $\mathrm{GH}$ or $\mathrm{PE}$, with no other comorbidities.

\section{Methods}

PE was diagnosed in patients with high blood pressure (24-h respiratory rate records) and i.e., new-onset proteinuria. The blood pressure was $\geq 140 / 90 \mathrm{mmHg}$ on two occasions that are at least four hours apart and proteinuria. In the absence of proteinuria, PE was diagnosed in women with hypertension with thrombocytopenia, impaired liver function, a new development of renal insufficiency, pulmonary edema, new-onset cerebral or visual disturbances, or uteroplacental dysfunction, including FGR.

FGR was diagnosed as fetal $A C / E F W<10^{\text {th }}$ percentile with a $\mathrm{PI}$ in $\mathrm{UA}>95^{\text {th }}$ percentile or a $\mathrm{PI}$ in $\mathrm{UA}>95^{\text {th }}$ percentile, or AC/EFW < $3^{\text {rd }}$ percentile, or the AEDF in the UA [13].

Neonatal wellbeing was recorded after birth (Apgar score). Parameters included in the analysis were neonatal body weight, week of gestation at birth and mode of delivery. The morphology was performed $24-48$ hours after delivery.

\begin{tabular}{|c|c|c|c|}
\hline Patients & $\begin{array}{l}\text { PE } \\
44\end{array}$ & $\begin{array}{l}\text { GH } \\
44\end{array}$ & $\mathbf{p}^{\mathbf{a}}$ \\
\hline Age (years, mean $\pm S D$ ) & $28 \pm 4$ & $30 \pm 4.6$ & 0.1 \\
\hline $\begin{array}{l}\text { Period of gestation } \\
\text { (median, } \min / \mathrm{max} \text { ) }\end{array}$ & $\begin{array}{l}36 \\
(27 / 41)\end{array}$ & $\begin{array}{l}39 \\
(35 / 42)\end{array}$ & $<0,001$ \\
\hline $\begin{array}{l}\text { Body mass index }\left(\mathrm{kg} / \mathrm{m}^{2}\right) \\
\text { (median, } \mathrm{min} / \mathrm{max} \text { ) }\end{array}$ & $\begin{array}{l}30 \\
(21 / 46)\end{array}$ & $\begin{array}{l}33 \\
(26 / 42)\end{array}$ & 7.013E-08 \\
\hline \multicolumn{4}{|l|}{ Parity } \\
\hline 0 & 36 & 34 & NT \\
\hline 1 & 7 & 7 & NT \\
\hline$>1$ & 1 & 3 & NT \\
\hline
\end{tabular}

${ }^{\mathrm{a}} \chi^{2}$ test of association or Mann-Whitney U-test comparing PE vs $\mathrm{GH}_{\text {; }}$ $\mathrm{PE}$ - preeclampsia; $\mathrm{GH}$ - gestational hypertension; NT — not tested 


\section{Statistics}

We used Statistica 11.0 (Statsoft). All comparisons were made with the nonparametric Mann-Whitney U test. Significance was set at $p<0.05$. Data were presented as medians with interquartile range and visualized with bar graphs. The tops of each bar indicate means, while lines represent $95 \%$ confidence intervals. The odds ratio (OR) was given as standard error and 95\% confidence interval. Significance was set at $\mathrm{p}<0.05$.

\section{RESULTS}

The time of delivery varied between the two groups. In our study PE mothers had delivered significant statistically earlier $(p<0.001)$ compared to $\mathrm{GH}$ as the median delivery time was $38(\min 27$; $\max 41)$ vs $40(\min 28 ; \max 42)$ week of pregnancy. Similarly, significant statistically earlier hospital administration time was noted for PE compared to GH mothers $(p<0.001)$, as it was $36(\min 27 ; \max 41)$ vs $39(\min 35$; max 42) week of pregnancy (Fig. 1). There was no statistical difference (between the duration of symptoms from the exacerbation of the disease to the delivery in the PE and GE group ( $\min 0$; $\max 6$ weeks) U test 0.167 (Fig. 1).

In this study, the clinical consequences of PE have been assessed thoroughly. The most important observation was that PE increased the risk of FGR. This was noticeable as the rate of FGR was $43 \%$ (18/42) for mothers with $P E$, compared to $8 \%(3 / 40)$ with gestational hypertension. The association was significant, $p<0.001$ but with moderate strength, Cramer's V $=0.40$. Thus, the risk of FGR increased nine times when PE occurred, as the odds ratio was 9.25 (Cl:2.46-34.83), $p=0,001$ (Fig. 2A).

PE was associated with FGR risk if delivery time was less than 34 weeks ( 11 mothers) compared to delivery time above 34 weeks (31 mothers). This was $82 \%(9 / 11)$ of FGR cases for $<34$ weeks, compared with $35 \%(8 / 23)$ of cases in the $>34$ group, $(p=0.001 ;$ Cramer's $V=0.50)$.
A

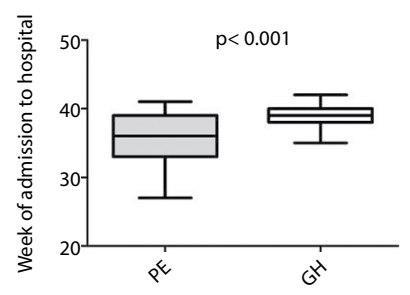

B

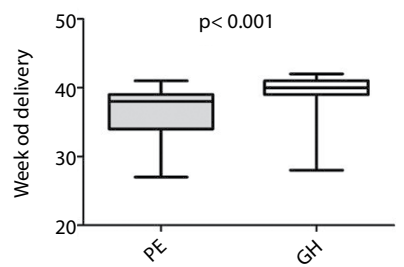

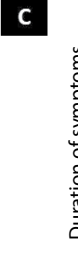

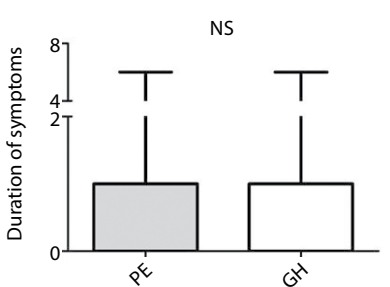

Figure 1. The clinical outcome of preeclampsia. A. the rate of fetal growth restriction in PE and GH groups, significantly higher in PE; B. significantly reduced child's body weight when the mother suffered from $\mathrm{PE}$ compared to $\mathrm{GH}$; $\mathbf{C}$. significantly higher rate of cesarean delivery by mothers suffering from PE compared to $\mathrm{GH}$; ${ }^{*}$ significant set to 0.05 ; 44 of patients both in PE and GH groups; NS — not significant; PE — preeclampsia; $\mathrm{GH}$ - gestational hypertension

A

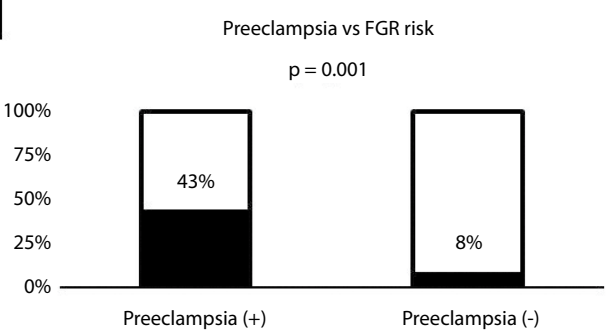

C

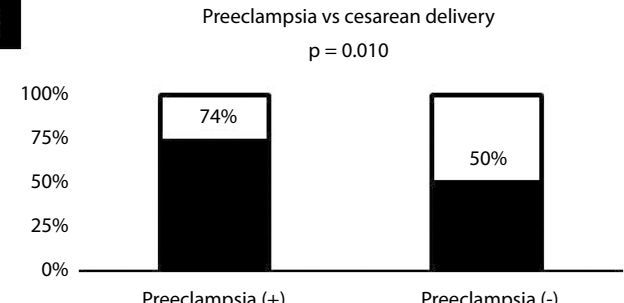

B

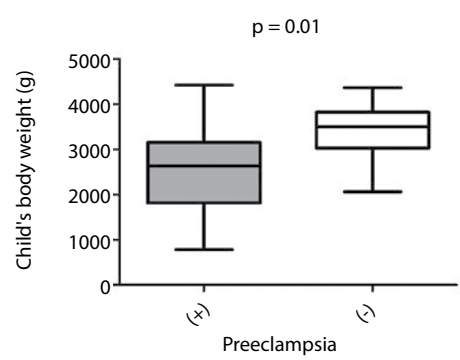

D

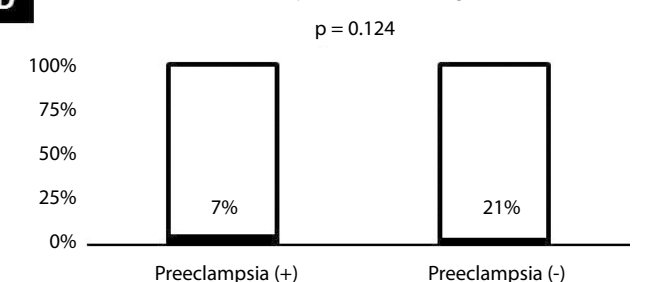

Figure 2. The clinical outcome of PE. A. the rate of fetal growth restriction in PE and GH groups, significant higher in PE; B. significant reduced child's body weight when mother suffered from PE compared to $\mathrm{GH} ; \mathbf{C}$. significant higher rate of caesarean delivery by mothers suffering from $\mathrm{PE}$ compared to $\mathrm{GH}$; D. significant higher rate of miscarriage by mothers suffering from $\mathrm{GH}$ compared to PE; * significant set to 0.05 ; 44 of patients both in PE and GH groups; FGR - fetal growth restriction 
In the GH group most of the patients were treated in monotherapy mode with the use of methyldopa (Dopegyt). The dosages used varied form $500 \mathrm{mg}$ per day to $2000 \mathrm{mg}$ per day. The second drug in patients qualified for a two drugs therapy was a calcium channel blocker. The drug most used was verapamil (Isoptin) (9 patients - 20.5\%). In the PE group we observed that patients were additionally treated with labetalol (8 patients - 18.1\%) Among the patients using labetalol the dose was between a $100 \mathrm{mg}$ and $200 \mathrm{mg}$ per day. Within the PE group there was a small percentage of patients treated with urapidil (Ebrantil/Tachyben)(6 patients - 13.6\%). In the group of patients treated with urapidil the authors noted a concurrent use of magnesium sulphate as eclampsia was a common condition among patients in this group.

The child's body weight was also lower when the mother suffered from PE $(p<0.001)$, as the median was $2630 \mathrm{~g}$ $(\min =780 ; \max =4420)$ compared to $3500 \mathrm{~g}(\min =2060$; $\max =4360$ ) with gestational hypertension (Fig. 2B). Further, PE was associated ( $p=0.010$, Cramer's $V=0.27$ ) with the type of delivery, as the cesarean section rate was $74 \%$ (31/42), compared to $50 \%$ (20/40) in the gestational hypertension group (Fig. 2C). This made it three-times higher the likelihood of delivery by caesarean section, as the odds ratio was 3.10 (Cl: 1.24-7.75), $\mathrm{p}=0.020$. Also, delivery time was significantly $(p<0.001)$ shortened to 38 weeks ( $\min =27$; $\max =41$ ), compared to 40 weeks $(\min =38$; $\max =42$ ) for gestational hypertension mothers. The rate of miscarriage was lower ( $p=0.050$; Cramer's V $=0.21$ ) for mothers with PE reaching 7\% (3/39) when compared with the opposite $24 \%(10 / 42)$. There was no distinction in median age for PE and $G H$ mothers ( $p=0,124$; Fig. 2D).

Of note is that the overall clinical status of neonates was proportioned despite the mother's PE. The sum of Apgar points in the first, and then the second to third minute, did not differ significantly, $p=0.370$ and 0.560 respectively. Up to 8 out of 42 of children born from mothers with PE had 4 to 7 Apgar points in the first minute and 34 had equal or higher than 8 points. In the second to third minute, the number of neonates that recorded equal or higher than 8 Apgar points increased to 40 , and only two had 4 to 7 points. In contrast, in the first minute 5 out of 42 of neonates, and then only one in the second to third minute, had 4 to 7 Apgar points when born from gestational hypertension mothers (Fig. 3A, 3B). The number of peripheral blood platelets and leucocytes wasn't reduced $(p=0.821$ and $p=0.534$ ) in infants when the mother suffered from $P E$, as it was $247000 / \mu \mathrm{L}(\min =29000$ / $/ \mu \mathrm{L} ; \max =585000 / \mu \mathrm{L})$ for platelets and $6.89 \times 10^{3} / \mu \mathrm{L}$ $\left(\min =12.27 \times 10^{3} / \mu \mathrm{L} ; \max =21.80 \times 10^{3} / \mu \mathrm{L}\right)$ leucocytes, compared to $252000 / \mu \mathrm{L}(\min =58000 / \mu \mathrm{L} ; \max =359000 / \mu \mathrm{L})$ for platelets and $7,04 \times 10^{3} / \mu \mathrm{L}\left(\min =12.90 \times 10^{3} / \mu \mathrm{L}\right.$; $\max =21.21 \times 10^{3} / \mu \mathrm{L}$ ) leucocytes in $\mathrm{GH}$ group.
Both BMI and bodyweight of mothers with PE was reduced significantly, $p=0.01$ and $p=0.010$ when compared to the gestational hypertension group. The median value was 33 ( $\min =26$; $\max =42$ ) for the gestational hypertension group and 30 ( $\min =21 ; \max =46$ ) for the former (Fig. 3C). Similarly, the body weight was $83 \mathrm{~kg}(\min =56$; $\max =132)$ for mothers with PE and $92 \mathrm{~kg}(\min =65 ; \max =125)$ for the latter (Fig. 3D).

\section{DISCUSSION}

Obstetricians managing women with preterm PE are faced with the challenge of balancing the need to achieve fetal maturation in utero with the risks to the mother and fetus of continuing the pregnancy longer. These risks include progression to eclampsia, development of placental abruption and HELLP syndrome.

In this study, despite a nine-times increased risk of FGR when PE occurred, the overall clinical status of neonates was comparable to the gestational hypertension group. Although PE may cause a low Apgar score, the need for NICU admission was not evident for our cohort [17]. The sum of Apgar points did not differ significantly. The only short-term complications were reduced child's body weight.

Another important observation was PE linked with the delivery by caesarean section. In our study, the risk of caesarean section was even three times higher as compared to the GH group. This was in line with previous reports that $25 \%$ of $\mathrm{PE}$ patients require delivery before 37 weeks About one-third of preterm births are medically indicated, $P E$ is the primary indication for iatrogenic preterm delivery [23, 24].

Maternal PE may affect other hematological parameters in the newborn. It may provoke neonatal thrombocytopenia (defined as a platelet count less than 150000/uL), occurring in the first three days of life, with most cases resolving by the tenth day [26]. Another hematological complication found in neonates is neutropenia (defined as neutrophil count $<500$ ). It has a variable course, lasting up to several weeks. A potential mechanism of PE mediated neutropenia is that uteroplacental insufficiency inhibits fetal bone marrow production of the myeloid line [26]. We did not confirm these observations as neither platelet nor leucocyte counts were affected in children born from PE mothers as compared to $\mathrm{GH}$ mothers.

Looking at mothers that developed PE, we have found that age was indistinct from the GH group, but what was interesting was that the BMI was lower for PE women. This is only partially in line with previous reports. Advanced maternal age $\geq 35$ years old at the time of delivery is associated with 1.2 to 3 -fold increased risk of developing PE [14-16]. Predictive probability of PE increases in pregnant women $\geq 35$ years old. The probability further increases rapidly in pregnant women $\geq 40$ years old. But the maternal 
A

Preeclampsia vs Apgar 1 (4-7 points)

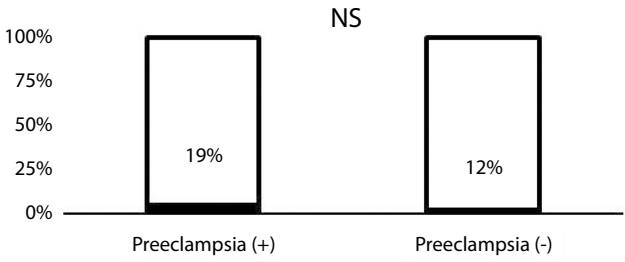

c

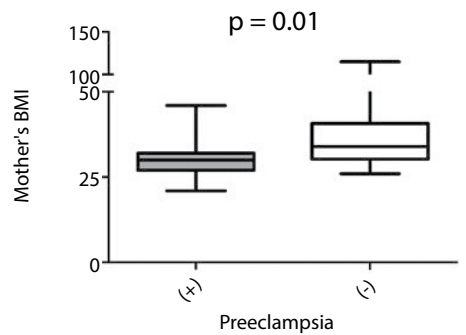

B

Preeclampsia vs Apgar 2/3 (4-7 points)

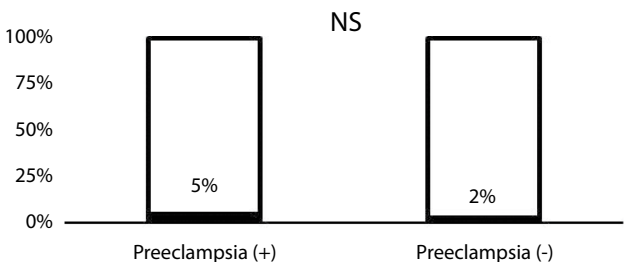

D

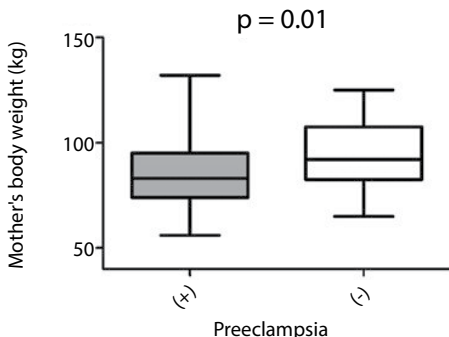

Figure 3. The clinical outcome of preeclampsia. A. no significantly different number of Apgar points (4 to 7) in first minute in PE and GH groups; B. no significantly different number of Apgar points (4 to 7) in second to third minutes in PE and GH groups; $\mathbf{C}$. significant lower BMI values of mothers suffering from PE compared to $\mathrm{GH}$; D. significant lower body weight values of mothers suffering from $\mathrm{PE}$ compared to $\mathrm{GH}$; * significant set to 0,$05 ; 44$ of patients both in PE and GH groups; NS — not significant; BMI — body mass indeks

age is not associated with increased risk of early-onset PE [17]. The increased risk of developing PE has been widely reported in nulliparous women. Duckitt K. et al. [18], showed the increased risk of PE 3-fold in nulliparous patients. Previous reports reported that $\mathrm{BMI} \geq 30 \mathrm{~kg} / \mathrm{m}^{2}$ has a 2 to 4 -fold higher risk for PE [19-21].

Several fetal complications are associated with PE, especially when the disease is severe or in early onset: FGR, oligohydramnios, IUFD, preterm delivery, non-reassuring FHR during delivery, low Apgar scores [22].

This paper shows that precise follow up of pregnant women developing hypertension during pregnancy may diminish the overall risk associated with this serious clinical condition. Despite the higher likelihood of FGR and other short-term complications like the risk of caesarian delivery and premature time of delivery, the overall clinical status of children born from mothers suffering from PE was normal. This is probably due to early diagnosis of hypertension and punctilious follow up of pregnant women.

In conclusion, the only definitive treatment of PE is to deliver the placenta and thus the neonate. Because of the two conflicting interests of pregnant woman and fetus, the time of delivery is one of the main challenges of $\mathrm{PE}$, especially in pregnant women with early-onset PE. Therefore, careful diagnosis and the proper assessment of the risk factors remains crucial in the management of the hypertensive diseases of pregnancy including the halting of the pregnancy.

\section{Funding}

The study was supported with the Polish NCN no. 2014/15/B/ /NZ5/03499.

\section{Conflict of interests}

There are no conflicts of interest to declare.

\section{REFERENCES}

1. Poon LC, Shennan A, Hyett JA, et al. The International Federation of Gynecology and Obstetrics (FIGO) initiative on pre-eclampsia: A pragmatic guide for first-trimester screening and prevention. Int J Gynaecol Obstet. 2019; 145(Suppl 1): 1-33, doi: 10.1002/ijgo.12802, indexed in Pubmed: 31111484.

2. Ronsmans C, Graham WJ. Lancet Maternal Survival Series steering group. Maternal mortality: who, when, where, and why. Lancet. 2006 368(9542): 1189-1200, doi: 10.1016/S0140-6736(06)69380-X, indexed in Pubmed: 17011946.

3. Kuklina EV, Ayala C, Callaghan WM. Hypertensive disorders and severe obstetric morbidity in the United States. Obstet Gynecol. 2009; 113(6): 1299-1306, doi: 10.1097/AOG.0b013e3181a45b25, indexed in Pubmed: 19461426.

4. Brown M, Magee L, Kenny L, et al. Hypertensive disorders of pregnancy. Hypertension. 2018; 72(1): 24-43, doi: 10.1161/hypertensionaha.117.10803.

5. Magee LA, Pels A, Helewa M, et al. Canadian Hypertensive Disorders of Pregnancy (HDP) Working Group. Diagnosis, evaluation, and management of the hypertensive disorders of pregnancy. Pregnancy Hypertens. 2014; 4(2): 105-145, doi: 10.1016/j.preghy.2014.01.003, indexed in Pubmed: 26104418.

6. Lowe SA, Bowyer L, Lust $K$, et al. Society of Obstetric Medicine of Australia and New Zealand. The SOMANZ guidelines for the management of hypertensive disorders of pregnancy 2014. Aust N Z J Obstet Gynaecol. 2015; 55(1): 11-16, doi: 10.1111/ajo.12253, indexed in Pubmed: 25308532.

7. Lisonkova $\mathrm{S}, \mathrm{Sabr} Y$, Mayer $\mathrm{C}_{1}$ et al. Maternal morbidity associated with early-onset and late-onset preeclampsia. Obstet Gynecol. 2014; 124(4): 
771-781, doi: 10.1097/AOG.0000000000000472, indexed in Pubmed: 25198279.

8. Bokslag A, van Weissenbruch M, Mol BW, et al. Preeclampsia; short and long-term consequences for mother and neonate. Early Hum Dev. 2016; 102: 47-50, doi: 10.1016/j.earlhumdev.2016.09.007, indexed in Pubmed: 27659865.

9. Mendola P, Mumford SL, Männistö TI, et al. Controlled direct effects of preeclampsia on neonatal health after accounting for mediation by preterm birth. Epidemiology. 2015; 26(1): 17-26, doi: 10.1097/EDE.0000000000000213, indexed in Pubmed: 25437315.

10. Vogel JP, Souza JP, Mori R, et al. Maternal complications and perinatal mortality: findings of the World Health Organization Multicountry Survey on Maternal and Newborn Health. BJOG: An International Journal of Obstetrics \& Gynaecology. 2014; 121(Suppl 1): 76-88, doi: 10.1111/1471-0528.12633.

11. von Dadelszen $P$, Magee LA. Preventing deaths due to the hypertensive disorders of pregnancy. Best Pract Res Clin Obstet Gynaecol. 2016; 36: 83-102, doi: 10.1016/j.bpobgyn.2016.05.005, indexed in Pubmed: 27531686.

12. Backes $\mathrm{CH}$, Markham $\mathrm{K}$, Moorehead $\mathrm{P}$, et al. Maternal preeclampsia and neonatal outcomes. J Pregnancy. 2011; 2011: 214365, doi: 10.1155/2011/214365, indexed in Pubmed: 21547086.

13. Saigal S, Doyle LW. An overview of mortality and sequelae of preterm birth from infancy to adulthood. Lancet. 2008; 371(9608): 261-269, doi: 10.1016/S0140-6736(08)60136-1, indexed in Pubmed: 18207020.

14. Khalil A, Syngelaki A, Maiz N, et al. Maternal age and adverse pregnancy outcome: a cohort study. Ultrasound in Obstetrics \& Gynecology. 2013; 42(6): 634-643, doi: 10.1002/uog.12494.

15. Lamminpää R, Vehviläinen-Julkunen $K$, Gissler $M$, et al. Preeclampsia complicated by advanced maternal age: a registry-based study on primi- parous women in Finland 1997-2008. BMC Pregnancy Childbirth. 2012; 12: 47, doi: 10.1186/1471-2393-12-47, indexed in Pubmed: 22687260.

16. Balasch J,GratacósE. Delayed childbearing. Current Opinion in Obstetrics \& Gynecology. 2012; 24(3): 187-193, doi: 10.1097/gco.0b013e3283517908.

17. Poon LCY, Kametas NA, Chelemen T, et al. Maternal risk factors for hypertensive disorders in pregnancy: a multivariate approach. J Hum Hypertens. 2010; 24(2): 104-110, doi: 10.1038/jhh.2009.45, indexed in Pubmed: 19516271.

18. Duckitt K, Harrington D. Risk factors for pre-eclampsia at antenatal booking: systematic review of controlled studies. BMJ. 2005; 330(7491): 565, doi: 10.1136/bmj.38380.674340.E0, indexed in Pubmed: 15743856.

19. Liu L, Hong Z, Zhang L. Associations of prepregnancy body mass index and gestational weight gain with pregnancy outcomes in nulliparous women delivering single live babies. Sci Rep. 2015; 5: 12863, doi: 10.1038/srep12863, indexed in Pubmed: 26242798.

20. Rahman MM, Abe SK, Kanda M, et al. Maternal body mass index and risk of birth and maternal health outcomes in low- and middle-income countries: a systematic review and meta-analysis. Obes Rev. 2015; 16(9): 758-770, doi: 10.1111/obr.12293, indexed in Pubmed: 26094567.

21. Wei YM, Yang HX, Zhu WW, et al. Risk of adverse pregnancy outcomes stratified for pre-pregnancy body mass index. J Matern Fetal Neonatal Med. 2016; 29(13): 2205-2209, doi: 10.3109/14767058.2015.1081167, indexed in Pubmed: 26427602.

22. Yücesoy $\mathrm{G}$, Ozkan $\mathrm{S}$, Bodur $\mathrm{H}$, et al. Maternal and perinatal outcome in pregnancies complicated with hypertensive disorder of pregnancy: a seven year experience of a tertiary care center. Arch Gynecol Obstet. 2005; 273(1): 43-49, doi: 10.1007/s00404-005-0741-3, indexed in Pubmed: 15834580. 